\title{
Design and Solution of a Surrogate Model for Portfolio Optimization Based on Project Ranking
}

\author{
Eduardo Fernandez, ${ }^{1}$ Claudia Gómez-Santillán, ${ }^{2}$ Laura Cruz-Reyes, ${ }^{2}$ \\ Nelson Rangel-Valdez, ${ }^{2,3}$ and Shulamith Bastiani ${ }^{2,4}$ \\ ${ }^{1}$ Faculty of Civil Engineering, Autonomous University of Sinaloa, Calz. de las Americas Nte., S/N, Cd. Universitaria, \\ 80013 Culiacán Rosales, SIN, Mexico \\ ${ }^{2}$ Postgraduate \& Research Division, National Mexican Technology/Madero Institute of Technology, Juventino Rosas 206, \\ 89440 Ciudad Madero, TAMPS, Mexico \\ ${ }^{3}$ CONACyT and Postgraduate \& Research Division, National Mexican Technology/Madero Institute of Technology, \\ Ciudad Madero, TAMPS, Mexico \\ ${ }^{4}$ National Mexican Institute of Technology/Tijuana Institute of Technology, Blvd. Alberto Limón Padilla y Av. ITR Tijuana, \\ S/N, Mesa Otay, 22500 Tijuana, BC, Mexico
}

Correspondence should be addressed to Claudia Gómez-Santillán; cggs71@hotmail.com

Received 14 June 2017; Revised 1 November 2017; Accepted 16 November 2017; Published 7 December 2017

Academic Editor: Giner Alor-Hernández

Copyright (C) 2017 Eduardo Fernandez et al. This is an open access article distributed under the Creative Commons Attribution License, which permits unrestricted use, distribution, and reproduction in any medium, provided the original work is properly cited.

\begin{abstract}
Characterizing the preferences of a decision maker in a multicriteria decision is a complex task that becomes even harder if the information available is limited. This paper addresses a particular case of project portfolio selection; in this case, the measures of project impacts are not assumed, and the available information is only projects' ranking and costs. Usually, resource allocation follows the ranking priorities until they are depleted. This action leads to a feasible solution, but not necessarily to a good portfolio. In this paper, a good portfolio is found by solving a multiobjective problem. To effectively address such dimensionality, the decision maker's preferences in the form of a fuzzy relational system are incorporated in an ant-colony algorithm. The Region of Interest is approached by solving a surrogate triobjective problem. The results show that the reduction of the dimensionality supports the decision maker in choosing the best portfolio.
\end{abstract}

\section{Introduction}

Resource allocation in institutions should address the proper distribution of a given budget among a set of available projects [1]. The type of projects can vary according to the area, and they might be involved in a wide range of tasks or activities, such as improving the skills of a professional sports team, the selection of $\mathrm{R} \& \mathrm{D}$ project portfolios in enterprises, the founding of projects by a government program, and supporting environmental regulations [2].

The construction of the best portfolio that accomplishes a certain balance among the selected projects and that is subject to a budget has been approached in the scientific literature (e.g., [3-8]). This problem can be defined as follows:

$$
\max _{x \in R_{F}}\left\{\left\langle z_{1}(x), z_{2}(x), \ldots, z_{p}(x)\right\rangle\right\},
$$

where $R_{F}$ is the space of feasible portfolios and $z(x)=$ $\left\langle z_{1}(x), z_{2}(x), \ldots, z_{p}(x)\right\rangle$ represents the functions $z_{i}$ that characterize the impact of a portfolio $x$ over the considered criteria.

Problem (1) has evolved in different particular cases. For example, recently, the Portfolio Selection Problem on a Set of Ordered Projects, or PSPSOP, has been reported to focus on the construction of a portfolio from a set of projects subject to a limited budget (cf. Bastiani et al. [9]). This case also involves a distinctive feature, which is that the only information available about the projects is their rank; that is, they are ordered according to the decision maker's (DM) preferences. The importance of the study of this problem arises from the fact that in most situations a DM prefers simple decision methods, and the decision process in such 
methods has conditions that involve limited resources such as time and information.

The task in PSPSOP is closely related to R\&D projects funded by enterprises; it can be seen in the discussion presented in Cooper et al. [10]. Particularly, in government organizations, $R \& D$ projects pursue the satisfaction of the citizenship, and, for this purpose, they try to identify programs that focus on the priorities of social sectors. The participatory budgeting aids in the definition of such priorities, and it combines the efforts of both citizens and government to establish them (cf. Fernandez and Olmedo [11]). With a proper definition of priorities, all that remains is the construction of a portfolio in which the costs of the projects adjust to the approved budget. This last task remains a challenge in the general case, and it has been studied from different approaches in the literature.

The construction of portfolios from projects that include information on ranking and costs is a problem that has been addressed in the scientific literature. The Ranking method is commonly used, and it involves the ordered selection of projects, previously ranked by priorities established by a DM, according to an available budget. This method has been criticized because the final portfolio that it creates only guarantees that the most important projects will be supported without considering a balance between the priorities and costs, which usually increases the number of supported projects and their impact in society (cf. $[12,13])$. Additionally, it could be possible that a DM becomes reluctant to follow such a construction if he considers that the ranking information is not reliable [14].

Bastiani et al. [9] propose a method to solve PSPSOP based on the cardinality and the discrepancies present in a portfolio, where cardinality refers to the total number of projects involved in it, and the term discrepancy is a concept that reflects the negative effect that is applied over the DM's thinking because one of the projects, when it is compared against others, seems to have merits that belong to the portfolio but it is not in it. The strategy balances the priorities and the number of projects in the final portfolio through a model that minimizes discrepancies concerning the ranking and costs and maximizes its cardinality, all of which are defined in ten objectives. Given that this model handles a large number of objective functions, this paper proposes a method to reduce the original many-objective problem to a surrogate one with three-objective functions. The reduced problem can be easily solved by an ant-colony algorithm that incorporates a knowledge mechanism (like the one proposed by Cruz et al. [15]).

This paper is organized into six sections. Section 2 presents a criticism of previous related approaches and describes the many-objective optimization model proposed by Bastiani et al. [9] for PSPSOP. Section 3 details the decision-support mechanisms used for the PSPSOP; this mechanism is based on DM preferences. Section 4 describes the metaheuristic optimization method. Finally, the experiments and results and conclusions are shown in Sections 5 and 6 , respectively.

\section{A Brief Outline of Previous Approaches}

According to the reviewed scientific literature, there are different approaches that solve PSPSOP with a lack of available information. The works of [16-18] are similar to Scoring and Ranking methods [10] or additive functions $[12,19]$ in that they prioritize projects according to a certain utility function to measure their importance. Alternatively, the use of proxy variables $[9,11,14]$ has offered versatile and satisfactory results that extend the information derived from a ranking of projects. However, none of those approaches offer a strategy that incorporates the DM's preferences, the key element in the present work that guides the construction of better solutions. Table 1 analyzes the state of the art related to the research proposed in these works. The first column presents the work, and the second column describes it.

From the information provided in Table 1, all the strategies followed to solve PSPSOP are based on ranking, costs, discrepancies, and cardinality. Our interest is focused on the definition and management of such elements given by the work of Bastiani et al. [9]. The remainder of this section briefly discusses their definition, but it is left up to the reader whether to get a deeper understanding of the concepts by reviewing the work in [9].

The model proposed by Bastiani et al. [9] defines three objectives per category plus one for the power indicator $P$. Hence, the number of proxy variables is $M+1$, where $M$ is the number of categories in the instance of PSPSOP that is solved. The model uses a reference portfolio $C_{\text {ref }}$ to reflect the DM's disappointment in combination with the statement " $\operatorname{rank}(x)$ is better than $\operatorname{rank}(y) \Rightarrow\left(y \in C_{\mathrm{ref}} \Rightarrow x \in C_{\mathrm{ref}}\right)$ " to define three types of discrepancies over a built portfolio $C$, which are initially based on the idea that a discrepancy occurs when in such a portfolio $a \in C_{\text {ref }}$ but $a \notin C$. These discrepancies are as follows: (a) weak discrepancy $n_{w k}$ that occurs when the budget of a project $x \in C_{\text {ref }}$, which is not in $C$, is much higher than the average budget in category $k$; (b) strong discrepancy $n_{s k}$ that occurs when the budget of a project $x \in C_{\text {ref }}$, which is not in $C$, is considerably higher than the average budget in category $k$; and (c) unacceptable discrepancy that occurs whenever a project $x \in C_{\text {ref }}$, which is not in $C$, has a budget that is not significantly higher than the average budget in category $k$, or the budget required by another project $y \in C$ that has a lower rank than $x$. The first two discrepancies, in combination with the cardinalities per category and the power of the set of objectives to the model, are proposed in [9]. The unacceptable discrepancy is used to constrain the feasible region of PSPSOP.

The model of Bastiani et al. [9], referred to as Bastiani's model in this work, succeeds in providing more information to a DM related to the construction of a particular portfolio. The success is derived from the specialization per category of the information derived from the ranking. However, due to this situation, the number of objectives involved in the model is not fixed, and it varies with the number of considered categories. This situation allows growth in the dimensionality, which in turn increases the difficulty in approximating the Region of Interest, that is, the zone in the Pareto frontier that fits a DM's preferences. For this reason, the question of 
TABLE 1: Optimization models for PSPSOP based on proxy variables.

Research work $\quad$ Description

To the best of our knowledge, this is the first published article that employs a set of proxy variables to solve PSPSOP. The

Fernandez et al. [14] model proposed works on discrepancies $D_{S}, D_{W}$ and cardinality $N_{C}$ of portfolios $C$; it is as follows:

$\min _{C \in R_{F}}\left(D_{S}, D_{W}\right), \max _{C \in R_{F}} N_{C}$.

This work also uses the cardinality of the portfolio $n_{p r}$ but it extends the types of discrepancies used in [14] to include the

Fernandez and Olmedo [11] set of absolute discrepancies $n_{a}$, which considers the absolute difference between the considered categories. The model is as follows:

$\max _{C \in R_{F}}\left\{\left\langle n_{p r}\right\rangle\right\}, \min _{C \in R_{F}}\left\{n_{a}, n_{s}, n_{w}\right\}$.

This work improves the multicriteria description of the portfolio qualities, provided by approaches such as [11, 14], through the incorporation of cardinality and discrepancy information per category. Additionally, it includes the power score $P$, an indicator of the number of high-rank projects in a

Bastiani et al. [9] portfolio. The proposed model is as follows:

$\underset{C \in R_{F}}{\text { Optimize }}\left\{\left\langle N_{1}, n_{\mathrm{wd} 1}, n_{\mathrm{sd} 1}, \ldots, N_{M}, n_{\mathrm{wd} M}, n_{\mathrm{sd} M}, P\right\rangle\right\}$,

where the power $P$ and cardinalities $N_{1}, N_{2}, \ldots, N_{M}$ are maximized and the discrepancies are minimized.

whether it is possible to keep the same level of information in the construction of a portfolio through an optimization approach but reduce the dimensionality remained open. Our work in this paper is dedicated to answering this question positively. The proposed approach is presented in the following section.

\section{Proposed Surrogate Model for PSPSOP}

According to Cruz et al. [15], metaheuristic approaches are a viable strategy to solve multiobjective optimization problems. These approaches rely on their ability to approximate the Pareto frontier to provide a set of solutions (or populations) that might satisfy complex constraints and objectives functions, a feature rarely shared with approaches based on mathematical programming, which normally yield a single solution and are limited to a narrower range of variations in the definition of an optimization problem.

During the optimization, it is possible to find some pitfalls derived from a problem with many objectives. One of the pitfalls arises if it is difficult to generate a proper population that lies on the Pareto frontier. Another pitfall corresponds to the increments in the number of Dominance Resistant Solutions, that is, solutions that are hard to dominate even though they are not Pareto optimal and are hard to take out of the final set of solutions. Another concern is related to the appropriate selection of one solution from the set given by metaheuristics. This situation leads to a process in which a human must perform a cognitive effort to choose the desired alternative. However, according to Miller [20], the human mind's capacity to handle information is diminished when it increases, for example, when the number of objectives that he/she must use to make a decision increases. All these drawbacks have been observed and/or discussed in several research studies, as shown in [21-24].

The high dimensionality of a problem can be addressed through the incorporation of preference information. According to the work in [15], a DM that is willing to provide his/her preferences aids in the localization of the Pareto frontier zone known as the Region of Interest (RoI); this zone can be understood as a relaxation of the feasible region defined by the multiobjective optimization problem that only covers solutions that are of interest for a DM. Several research papers, such as the one by Fernandez et al. [25], present strategies that address multiobjective problems using surrogate models that approximate the RoI. The remaining part of this section presents the adaptation of such model to solve the PSPSOP.

The research work in [25] proposes a surrogate model based on the credibility index $\sigma(x, y)$ of the statement " $x$ is at least as good as $y$." The value of $\sigma(x, y)$ is computed using the method ELECTRE (cf. $[26,27])$, and it is integrated into a relational system of preferences (as described by Roy [26]) to model the DM preferences. The preferences defined in [27] for pairs of alternatives $x$ and $y$ are as follows: (1) strict preference $x P y$; (2) indifference $x I y$; (3) weak preference $x Q y$; (4) incomparability $x R y$; and (5) $k$-preference $x K y$.

To define a surrogate model for PSPSOP, this work uses the net flow score defined through the objectives of Bastiani's model, as shown in Table 2. The net flow score, denoted $F_{n}(x)$ or NF, was introduced in Fernandez et al. [25] as a measure to enhance preference information towards a better characterization of the DM preferences in the nonstrictly outranked set, denoted by NS. Based on the elements $y \in$ NS, 


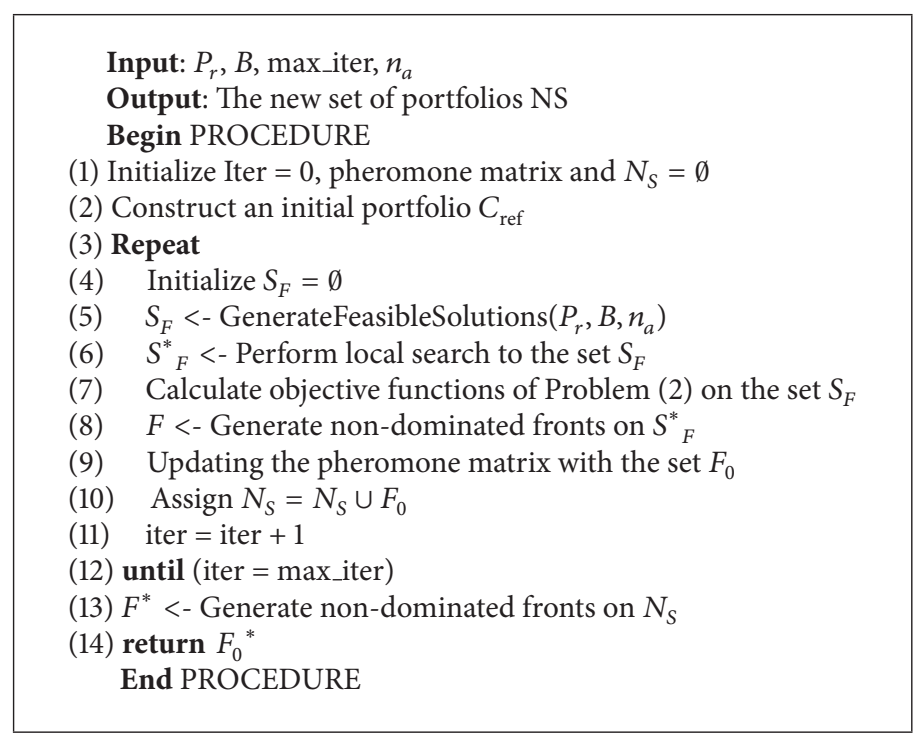

Algorithm 1: ACO-SOP.

TABLE 2: Computation of the net flow score.

\begin{tabular}{ll}
\hline Set/measure & Conditions \\
\hline$S$ & $S(O, x)=\{y \in O \mid y P x\}$. \\
NS & $\mathrm{NS}(O)=\{x \in O \mid S(O, x)=\varnothing\}$. \\
$W$ & $W(O, x)=\{y \in \mathrm{NS}(O) \mid y Q x \vee y K x\}$ \\
& $F_{n}(x)=\sum_{y \in \mathrm{NS}(O) \backslash\{x\}}[\sigma(x, y)-\sigma(y, x)]$, \\
Net flow score $(\mathrm{NF})$ & where $F_{n}(x)>F_{n}(y)$ denotes a certain \\
& preference of $x$ over $y$ \\
$F$ & $F(O, x)=\left\{y \in \mathrm{NS}(O) \mid F_{n}(y)>F_{n}(x)\right\}$ \\
\hline
\end{tabular}

the following sets are defined: (a) the set $S$ of alternatives $y$ that strictly outrank $x$; (b) the set $W$ of portfolios $y$ that weakly outrank a given portfolio $x$; and (c) the set $F$ of alternatives $y$ with greater net flow score. All the previous elements are defined in Table 2, where $O$ is the set of feasible portfolios.

The combination of the net flow score $F_{n}(x)$ with the definition of the sets $S$ and $W$ allows the formulation of the optimization problem defined in

$$
\min _{x \in O}\{\langle|S(O, x)|,|W(O, x)|,|F(O, x)|\rangle\} .
$$

With the previous problem, it is demonstrated that Bastiani's model can be mapped to problem (2) independently of the original objective space dimension. The approach by Fernandez et al. [25] was successfully applied to Problem 1 in [15]. Combined with an ant-colony metaheuristic, the approach in Bastiani et al. [9] is extended here to solve PSPSOP thru the optimization model defined in problem (2). To summarize, the contribution of this work is the solution of PSPSOP with lack of information using a surrogate objective model (which is of smaller dimension than those reported in previous approaches) and the incorporation of DM's preferences in the search process to approximate the RoI. The details of the algorithm and each component of the ACO-SOP are presented in the next section.

\section{An Ant-Colony Optimization Algorithm}

This work proposes an approach based on an ant-colony optimization, denoted by ACO-SOP (Ant-Colony Optimization for Solving Portfolio Problems with Ordinal Information about Projects). The approach takes ideas from Dorigo's ACS [28], Cruz et al. [15], and Bastiani et al. [9] to solve PSPSOP, first searching in the wider feasible region defined by Bastiani's model and then searching in the smaller space defined by the model in problem (2).

The core strategy of ACO-SOP uses the pheromone representation, selection rule, and local search function defined in the algorithm ACO-SPRI proposed by Bastiani et al. [9], with a small variation in the construction of the nondominated fronts used in their computations. The general idea of ACO-SOP is depicted in Algorithm 1. The algorithm is characterized by five elements: (a) the initialization function; (b) the construction of a feasible set of solutions; (c) the improvement phase based on local search; (d) the construction of nondominated fronts; and (e) the updating of the pheromone matrix. These elements are briefly defined in this section (cf. Bastiani et al. [9] for further details).

The algorithm ACO-SOP requires as input the set $P_{r}$ of ranked projects, the budget $B$, the maximum number of iterations max_iter, and the maximum number of ants $n_{a}$. Inside this algorithm, the initialization function constructs the reference portfolio $C_{\text {ref }}$ (see Line (2)); it is done by the ordered selection of projects from $P_{r}$ by rank and in agreement with the available budget $B$ (ties are broken arbitrarily).

The phase of construction of feasible solutions (see Line (5)) involves the construction of one portfolio by each of the $n_{a}$ ants. Each ant starts with an empty portfolio $C$ and adds to it one project at a time based on the budget, global knowledge 


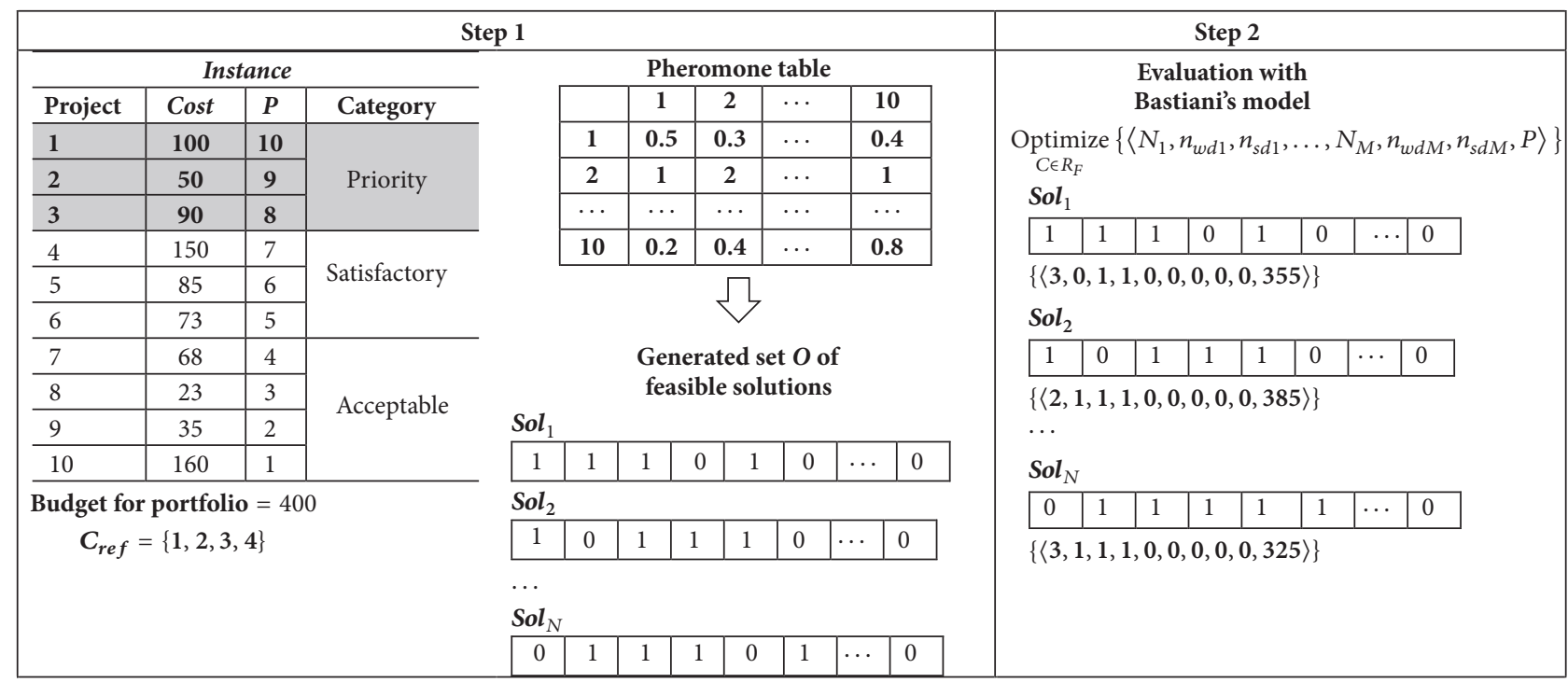

\begin{tabular}{|c|c|c|c|c|c|c|c|c|}
\hline \multicolumn{8}{|c|}{ Step 3} & Step 4 \\
\hline \multicolumn{8}{|c|}{$\begin{array}{l}\text { Apply local search in feasible } \\
\text { solutions }\end{array}$} & $\begin{array}{l}\text { Input: } \\
\operatorname{Sol}_{1}\{\langle 4,0,0,1,0,0,0,0,0,390\rangle\}\end{array}$ \\
\hline \multicolumn{8}{|c|}{ Sol $_{1}$} & $\begin{array}{l}\mathrm{Sol}_{2}\{\langle 3,1,1,2,0,1,0,0,0,390\rangle\} \\
\ldots\end{array}$ \\
\hline \multicolumn{8}{|c|}{$\langle 4,0,0,1,0,0,0,0,0,390\rangle$} & $\operatorname{Sol}_{N}\{\langle 3,2,2,1,1,0,0,0,0,370\rangle\}$ \\
\hline \multicolumn{8}{|c|}{$\mathrm{Sol}_{2}$} & Evaluation with \\
\hline 1 & 0 & 1 & 1 & 1 & 1 & & 0 & surrogate model \\
\hline \multicolumn{8}{|c|}{$\begin{array}{l}\{\langle 3,1,1,2,0,1,0,0,0,390\rangle\} \\
\cdots\end{array}$} & $\min _{x \in O}\{\langle|S(O, x)|,|W(O, x)|,|F(O, x)|\rangle\}$ \\
\hline \multicolumn{8}{|c|}{$\operatorname{Sol}_{N}$} & $\operatorname{Sol}_{1}\{\langle 0,0,1\rangle\}$ \\
\hline 0 & 0 & 1 & 1 & 1 & 1 & \begin{tabular}{|l|}
$\cdots$ \\
\end{tabular} & 1 & $\operatorname{Sol}_{2}\{\langle 1,1,2\rangle\}$ \\
\hline \multicolumn{8}{|c|}{$\langle 3,2,2,1,1,0,0,0,0,370\rangle$} & $\operatorname{Sol}_{N}\{\langle 1,1,0\rangle\}$ \\
\hline
\end{tabular}

FIGURE 1: Schematic example of construction process of ACO-SOP.

derived from the pheromone matrix, and local knowledge obtained from the preferences of the DM, as defined in the selection rule by Bastiani et al. [9]. At the end of this phase, a set $S_{F}$ of feasible portfolios is delivered.

Following the process of forming $S_{F}$, the algorithm ACOSOP performs a local search over each $C$ in $S_{F}$, just as it is done by Bastiani et al. [9] in their local search scheme. The algorithm creates a new portfolio $C^{\prime}$ new with every combination derived from the inclusion and/or exclusion of $v$ projects of $P_{r}$ chosen at random. Each portfolio $C^{\prime}{ }_{\text {new }}$ is repaired such that constraints in rank and budget are held. Finally, the best portfolio $C^{*}{ }_{n e w}$ is chosen as the local search improvement for $C$. The union of all the new portfolios $C^{*}$ new will form the new set of feasible portfolios $S_{F}^{*}$.

The construction of nondominated fronts (see Line (8)) is based on the objectives defined in problem (2). The output of the algorithm in this phase is the set of fronts $F=$ $\left\{F_{0}, F_{1}, \ldots, F_{k}\right\}$ obtained from $S^{*}$. Each set $F_{i}$ is composed of a subset of portfolios in $S_{F}^{*}$ that are exclusively dominated by exactly $i$ portfolios, where $0 \leq i \leq k$ (following the dominance criterion established in Cruz et al. [15]). Instead of computing the dominance from the objectives of Bastiani's model, this work uses the objectives of problem (2), a key feature in this research because it makes it more manageable for algorithms to address large dimensions.

Finally, the last and most important element that defines ACO-SOP is the pheromone matrix, and the process to construct it and update it is described herein. The bidimensional matrix $\tau$ represents the knowledge that the ants have gained during the construction of portfolios. They represent that knowledge in the form of pairs of projects $(i, j)$ and the gain of having them together in the same portfolio (denoted as $\tau_{i, j}$ ). The portfolios $S_{F}^{*}$ constructed by the ants at each iteration are used to update $\tau$ (see Line (9)) based on the number of fronts $k$ constructed and in the front where each portfolio is found. This strategy and the one incorporated to prevent premature convergence are detailed in Bastiani et al. [9].

The algorithm ACO-SOP accumulates in $N_{S}$ the front $F_{0}$ constructed at each iteration (see Line (10)). Then, using the last set $N_{S}$ it forms the final set of fronts $F^{*}$ and returns as its solution the front $F^{*}$. Figure 1 presents a 
TABLE 3: Parameters of the outranking model.

\begin{tabular}{|c|c|c|c|c|c|c|c|c|c|}
\hline \multicolumn{10}{|c|}{ Values of the preference model } \\
\hline \multirow{2}{*}{\multicolumn{2}{|c|}{$W$}} & \multirow{2}{*}{\multicolumn{2}{|c|}{$\begin{array}{c}\mathrm{Q} \\
\text { Indifference thresholds } \\
\end{array}$}} & \multirow{2}{*}{\multicolumn{2}{|c|}{$\begin{array}{c}P \\
\text { Strict preference threshold }\end{array}$}} & \multirow{2}{*}{\multicolumn{2}{|c|}{$\begin{array}{c}U \\
\text { Pre-veto threshold }\end{array}$}} & \multirow{2}{*}{\multicolumn{2}{|c|}{$\begin{array}{c}\text { V } \\
\text { Veto threshold } \\
\end{array}$}} \\
\hline & & & & & & & & & \\
\hline$\overline{W_{1}}$ & 0.21 & $Q_{1}$ & 0 & $P_{1}$ & 1 & $U_{1}$ & 2 & $V_{1}$ & 3.5 \\
\hline$W_{2}$ & 0.14 & $Q_{2}$ & 0 & $P_{2}$ & 1 & $U_{2}$ & 2.5 & $V_{2}$ & 5.5 \\
\hline$W_{3}$ & 0.21 & $Q_{3}$ & 0 & $P_{3}$ & 1 & $U_{3}$ & 1.5 & $V_{3}$ & 3 \\
\hline$W_{4}$ & 0.14 & $Q_{4}$ & 0 & $P_{4}$ & 1 & $U_{4}$ & 8 & $V_{4}$ & 11.5 \\
\hline$W_{5}$ & 0.05 & $Q_{5}$ & 0 & $P_{5}$ & 1 & $U_{5}$ & - & $V_{5}$ & - \\
\hline$W_{6}$ & 0.12 & $Q_{6}$ & 0 & $P_{6}$ & 1 & $U_{6}$ & - & $V_{6}$ & - \\
\hline$W_{7}$ & 0.04 & $Q_{7}$ & 0 & $P_{7}$ & 1 & $U_{7}$ & - & $V_{7}$ & - \\
\hline$W_{8}$ & 0.01 & $Q_{8}$ & 0 & $P_{8}$ & 2 & $U_{8}$ & - & $V_{8}$ & - \\
\hline$W_{9}$ & 0.04 & $Q_{9}$ & 0 & $P_{9}$ & 1 & $U_{9}$ & - & $V_{9}$ & - \\
\hline$W_{10}$ & 0.04 & $Q_{10}$ & 0.5 & $P_{10}$ & 1.5 & $U_{10}$ & - & $V_{10}$ & - \\
\hline \multicolumn{10}{|c|}{ Credibility threshold $\lambda=0.67$} \\
\hline \multicolumn{10}{|c|}{ Unacceptability threshold $\gamma=0.20$} \\
\hline
\end{tabular}

Note. Veto power is not allowed in criteria 5-10.

brief example of the construction process of ACO-SOP. Step 1 shows how the instance information is transformed with the aid of the pheromone matrix into a set of $N$ feasible solutions $O=\left\{\mathrm{Sol}_{1}, \mathrm{Sol}_{2}, \ldots, \mathrm{Sol}_{N}\right\}$, where each solution $\mathrm{Sol}_{i}$ is binary vector representing the projects in a portfolio. Step 2 shows the computed values of the 10 objectives from Bastiani's model for each solution. After that, in step 3, ACOSOP shows an improved set of $N$ solutions from the local search. Finally, step 4 presents the transformation of $O$ into the surrogate model, that is, a set $O$ with the objective values defined in problem (2). To exemplify the transformation, let us take the values of the solution $x=\operatorname{Sol}_{1}=\langle 0,0,1\rangle$, which indicate that there is no other solution $y=\operatorname{Sol}_{i}$, for $i \neq j$, that is strictly or weakly preferred over $x$; that is, there is no $y$ such that $y P x$ or $y Q x$ or $y K x$, and there is only one solution that has greater net flow score than $x$; that is, $F_{n}(y)>F_{n}(x)$, taking into account the fact that $y$ is in the nonstrictly outranked set $\mathrm{NS}(\mathrm{O})$.

The following section presents the experimental design performed to evaluate the performance of ACO-SOP when solving problem (2). The following section also shows the versatility of the approach to tackle cases of large dimensions.

\section{Solving Some Computer Experiments}

In this section, we develop a set of experiments to verify the validity of and to validate the advantages gained by our solution method, especially addressing problems with many objectives. Based on these goals, the exposure and resolution of two different cases of study are presented in the following paragraphs.

To test the performance of ACO-SOP, the algorithm was implemented using the programming language Java. The experimental design was made using a computer with an Intel Core i7 $2.8 \mathrm{GHz}$ CPU, $4 \mathrm{~GB}$ of RAM, and Mac OS $\mathrm{X}$ Lion 10.7 .5 (11G63b) as operating system. The instance used for the experimentation is the one defined in Bastiani et al. [9]. The tuning process involved selecting different combinations of values similar to those previously reported for these parameters. According to the results of the finetuning process, the best configuration of values was as follows: tot_iter $=200$ iterations, $n_{a}=300$ ants, $w=0.63$, $\eta=0.1, \rho=0.9, \alpha_{1}=0.65$, and $\alpha_{2}=0.75$. These parameter values were used to obtain the experimental results reported in this section. The incorporation of preferences through the outranking model is basic in order to map the problem in Bastiani et al. [9] into problem (2). The parameters of the outranking model were set as provided in Table 3.

The ACO-SOP strategy requires, besides the ranking information and costs of the PSPSOP instance, the definition of a reference portfolio $C_{\text {ref }}$ and the definition of the projects' categories. The heuristic followed to construct $C_{\text {ref }}$ is based on the traditional Scoring and Ranking method and takes the projects one at a time in order of their rank until the available budget is consumed. The categories considered during the experiment were (1) priority, (2) satisfactory, and (3) acceptable, and the projects were distributed uniformly among them based on their ranks.

5.1. Analysis of Results. The results from the experiments performed on the proposed approach (ACO-SOP) are analyzed from three points of view. The first analysis involves the quality of the solutions provided by ACO-SOP in relation to their meaning to a DM and his/her preferences. The second and third analyses study the performance of ACO-SOP in comparison with a similar approach of the state of the art. These experiments differ on the quality indicator used during the comparison. Let us indicate that the considered budget was 2,500 units and that the reference portfolio $C_{\text {ref }}$ is formed by the first 22 best ranked projects in the instance taken from Bastiani et al. [9].

The indicators used to measure the results from the experiment were $I_{1}, I_{2}$, and $I_{3}$. The indicator $I_{1}=$ $\{|S(O, x)|,|W(O, x)|,|F(O, x)|\}$ uses the three objectives associated with the preference information derived from the proposed surrogate model (see problem (2)). The indicator 
TABLE 4: Nonstrictly outranked solutions.

\begin{tabular}{|c|c|c|c|c|c|c|c|c|c|c|c|c|c|c|}
\hline \multirow{2}{*}{$\begin{array}{l}\text { Solution } \\
x\end{array}$} & \multicolumn{3}{|c|}{$I_{1}$} & \multicolumn{11}{|c|}{$I_{2}$} \\
\hline & $|S(O, x)|$ & $|W(O, x)|$ & $|F(O, x)|$ & $N_{1}$ & $n_{\mathrm{wd} 1}$ & $n_{\mathrm{sd} 1}$ & $\mathrm{~N}_{2}$ & $n_{\mathrm{wd} 2}$ & $n_{\mathrm{sd} 2}$ & $N_{3}$ & $n_{\mathrm{wd} 3}$ & $n_{\text {sd } 3}$ & $P$ & Card \\
\hline$A C O-S O P_{1}$ & 0 & 0 & 0 & 27 & 2 & 0 & 1 & 0 & 0 & 0 & 0 & 0 & 85.10 & 28 \\
\hline ACO-SOP 2 & 0 & 0 & 1 & 27 & 4 & 0 & 6 & 0 & 0 & 0 & 0 & 0 & 81.03 & 33 \\
\hline $\mathrm{ACO}-\mathrm{SOP}_{3}$ & 0 & 2 & 2 & 27 & 2 & 1 & 3 & 0 & 0 & 0 & 0 & 0 & 83.5 & 30 \\
\hline
\end{tabular}

TABLE 5: Solutions suggested in [11] and the two best solutions to the problem in Bastiani et al. [9].

\begin{tabular}{lccccccccccc}
\hline Solution & $N_{1}$ & $n_{\mathrm{wd} 1}$ & $n_{\mathrm{sd} 1}$ & $N_{2}$ & $n_{\mathrm{wd} 2}$ & $n_{\mathrm{sd} 2}$ & $N_{3}$ & $n_{\mathrm{wd} 3}$ & $n_{\mathrm{sd} 3}$ & $P$ & Card $(C)$ \\
\hline Sol $_{1}$ & 24 & 0 & 0 & 0 & 0 & 0 & 0 & 0 & 0 & 87.62 & 24 \\
Sol $_{2}$ & 25 & 0 & 0 & 0 & 0 & 0 & 0 & 0 & 0 & 86.96 \\
ACO-SOP $_{1}$ & 27 & 2 & 0 & 1 & 0 & 0 & 0 & 0 & 0 & 85.10 & 25 \\
ACO-SOP $_{2}$ & 27 & 4 & 0 & 6 & 0 & 0 & 0 & 0 & 0 & 81.03 & 33 \\
\hline
\end{tabular}

$I_{2}=\left\{N_{1}, n_{\mathrm{wd} 1}, n_{\mathrm{sd} 1}, N_{2}, n_{\mathrm{wd} 2}, n_{\mathrm{sd} 2}, N_{3}, n_{\mathrm{wd} 3}, n_{\mathrm{sd} 3}, P\right\}$ uses the ten objectives associated with ranking and derived from the Bastiani model (see Section 2). Finally, the indicator $I_{3}$ is the content of the portfolio, that is, the projects that are included in it.

The first analysis involved the solution of the instance with ACO-SOP, and its results are summarized in Table 4. This table provides the values of the indicators $I_{1}$ and $I_{2}$ computed from the three best nonstrictly outranked solutions that were consistently obtained in 30 runs (the average computer time required for this experiment was 130 minutes). The solutions ACO-SOP $1, A C O-S_{2}$, and ACO-SOP 3 of Table 4 are ordered according to the indicator $I_{1}$; in this ordering, ACO-SOP 1 is the best because it reduced all the values of the indicator to zero, while $\mathrm{ACO}-\mathrm{SOP}_{2}$ did it only for the metrics $|S(O, x)|$ and $|W(O, x)|$ but it improves ACO$\mathrm{SOP}_{3}$ in the last one, $|F(O, x)|$. This relation of order is associated with the DM's preferences, and evidence of that can be supported by analyzing the values of the indicator $I_{2}$, regarding the number of projects involved in the solutions.

First, it is possible to observe a great level of dissatisfaction in the DM when the provided solutions have strong discrepancies (see value $n_{\text {sd } 1}$ of $\mathrm{ACO}-\mathrm{SOP}_{3}$ ), because it means that some important projects that are in the reference portfolio $C_{\text {ref }}$ are left out of the solution when they could have been included in place of others that were less relevant. Second, the DM could have a low level of dissatisfaction if the solutions only have weak discrepancies (see value $n_{\mathrm{wd} 1}$ of ACO$\mathrm{SOP}_{2}$ ), because it means that only a rather small number of important projects had been left out. Third, an almost null level of dissatisfaction can be noted in the DM when the solution involved a small number of weak discrepancies and a high power (see values $n_{\mathrm{wd} 1}$ and $P$ of ACO-SOP ${ }_{1}$ ), because this is an indicator that an adequate number of high-ranked projects are in the solution.

The second analysis compares the two best solutions ACO-SOP 1 and ACO-SOP 2 against the solutions Sol $_{1}$ and $\mathrm{Sol}_{2}$, as reported in [11]. Table 5 summarizes these results, with each of its columns representing a metric used by indicator $I_{2}$. Observe that the lack of discrepancies on Sol $_{1}$ and $\mathrm{Sol}_{2}\left(\right.$ see $n_{\mathrm{wd} 1}, n_{\mathrm{sd} 1}, n_{\mathrm{wd} 2}, n_{\mathrm{sd} 2}, n_{\mathrm{wd} 3}$, and $\left.n_{\mathrm{sd} 3}\right)$ could allow the DM to make a decision using only two criteria, the power $P$ and the cardinality $N_{1}$; this is a convenient situation for a DM that willingly accepts a solution with only highranked projects, or with a large cardinality, but does not care about their balance (because high values in $P$ are normally associated with small cardinalities and high-ranked but costly projects). But what happens if the DM has a different point of view? For example, how are these solutions affected if, for the DM, the objectives are not equally important? The latter case involves considering solutions, such as ACO-SOP 1 and ACO-SOP ${ }_{2}$, with an acceptable level of discrepancies and a better balance between $P$ and the cardinalities (e.g., $N_{1}$ and $N_{2}$ ); these solutions slightly increase the discrepancies (as in metric $n_{\mathrm{wd} 1}$ ). Hence, while approaches such as the ones presented in [11] or in Bastiani et al. [9] succeeded in managing problems with limited information, they do not include DM preferences. This analysis showed evidence that the solutions provided by the proposed model allow the inclusion of DM's preferences through the parameters of the outranking model (see Table 3 ). The preference model can be used to redefine what is acceptable or not as a solution for a DM; for example, the solutions $\mathrm{Sol}_{1}$ and $\mathrm{Sol}_{2}$ do not satisfy the DM's preferences in Table 3 because they have unacceptable discrepancies that cause great discomfort to the DM; this situation is detailed in the following analysis.

Table 6 allows the analysis of the configurations of the portfolios obtained from $\mathrm{Sol}_{1}, \mathrm{Sol}_{2}, \mathrm{ACO}-\mathrm{SOP}_{1}$, and ACO$\mathrm{SOP}_{2}$; it shows the projects in each solution and in bold those in Category 1. One important difference between these solutions is the situation of Project $20\left(\right.$ or $\left.\mathrm{Pj}_{20}\right)$; while it appears on the portfolio of ACO-SOP 1 and ACO-SOP 2 , it does not in $\mathrm{Sol}_{1}$ and $\mathrm{Sol}_{2}$. The relevance of this project is that it belongs to the reference portfolio $C_{\text {ref }}=\{1,2,3, \ldots, 22\}$, and the fact that it does not appear in $\mathrm{Sol}_{1}$ and $\mathrm{Sol}_{2}$ constitutes an unacceptable discrepancy for the DM characterized in Table 3 .

To explain this situation, let us note that $P j_{20}$ is in Category 1 and has an average cost of $\operatorname{Cost}\left(P j_{20}\right)=97.5$ and the projects of $\mathrm{Sol}_{1}$ that are in the same category 
TABLE 6: Projects of the portfolios created from solutions $\mathrm{Sol}_{1}, \mathrm{Sol}_{2}, \mathrm{ACO}-\mathrm{SOP}_{1}$, and ACO-SOP 2 .

\begin{tabular}{|c|c|c|}
\hline Solution & Projects in the portfolio & $\operatorname{Avg}\left(\mathrm{Cat}_{1}\right)$ \\
\hline $\mathrm{Sol}_{1}$ & $\{1,2,3,4,5,6,7,8,9,10,11,12,13,14,15,16,17,18,19,21,24,25,26,27\}$ & 102.19 \\
\hline $\mathrm{Sol}_{2}$ & $\{1,2,3,4,5,6,7,8,9,10,11,12,13,14,15,16,17,18,19,21,24,25,26,27,28\}$ & 99.55 \\
\hline ACO-SOP & $\{1,2,3,4,5,6,7,8,9,10,11,12,13,14,15,16,17,18,20,22,25,26,27,28,29,30,33,34\}$ & 91.29 \\
\hline $\mathrm{ACO}-\mathrm{SOP}_{2}$ & $\{1,2,3,4,5,6,7,8,9,10,11,12,13,14,15,16,17,18,20,25,26,27,28,29,30,31,32,33,34,35,36,37,38,39\}$ & 85.31 \\
\hline
\end{tabular}

have an average cost of $\operatorname{Avg}\left(\mathrm{Cat}_{1}\right)=102.19$. Hence, the unacceptability level of $P j_{20}$ is $\operatorname{UNAc}\left(P j_{20}\right)=\left(\operatorname{Cost}\left(P j_{20}\right)-\right.$ $\left.\operatorname{Avg}\left(\mathrm{Cat}_{1}\right)\right) / \operatorname{Avg}\left(\mathrm{Cat}_{1}\right)=-0.04$. The value of $\operatorname{UNAc}\left(P j_{20}\right)$ is smaller than the unacceptability threshold $\gamma=0.20$ defined for the DM; that is, the projects in the portfolio are too costly to exclude $P j_{20}$ from $C_{\text {ref }}$ and possibly some others, a situation that occurs with $\mathrm{Sol}_{1}$ and $\mathrm{Sol}_{2}$ and that generally does not satisfy the DM's preferences. Such solutions where the DM's preferences are not considered can be constructed using approaches from $[9,11]$, but the present approach overcomes it by including preferences during the search process; as a result, the solutions contain portfolios more convenient for the DM, because they include an increment in the number of projects of up to $20 \%$, with the guarantee that this still satisfies his/her preferences.

To summarize, this section has presented the results from the experiment to analyze the quality of the solutions provided by ACO-SOP and their robustness. The robustness was demonstrated by providing evidence that ACO-SOP provides solutions of the same quality as the other approaches, whenever they satisfy the DM's preferences; evidence of this is provided in Table 4, where solutions of similar quality are presented, solutions that are in the RoI for a DM. In general, whenever a solution fits the interest of the DM expressed in the parameter values of the preference model, this solution can be found in the search space, showing that the present approach maintains the same level of information. Finally, the lack of preferences on approaches such as [11] or [9] can lead to the construction of solutions that are not in the RoI for a $\mathrm{DM}$, that is, solutions the DM is not interested in.

\section{Some Conclusions}

This paper proposes a knowledge-based decision-support system for the Portfolio Selection Problem on a Set of Ordered Projects. This problem commonly has a lack of available information, which is why the incorporation of knowledge mechanisms in the solution strategies can improve the quality of the solutions.

This work is a crucial refinement from a recent proposal that modeled the DM's attitude through a many-objective optimization problem. The high dimensionality of this problem is a great concern for metaheuristic approaches in generating an acceptable approximation to the Pareto frontier, and it is also a great concern for the DM in making the final decision due to human cognitive limitations. Here, we have presented a method that incorporates the DM's preferences through a fuzzy outranking-based relational model. This allows mapping the original many-objective problem into a surrogate three-objective problem. With this transformation, the process of identifying the Region of Interest (the preference privileged zone of the Pareto frontier) becomes easy. Another advantage of the proposal is to be very robust with respect to an increasing number of objective functions. Robustness is important to make a finer representation of the DM's preferences, including project synergic effects. So this contribution should be significant in this particular field of project portfolio optimization.

The strongly nonlinear surrogate problem is solved by using an ant-colony algorithm. This algorithm includes a knowledge and learning mechanism in the form of a pheromone matrix and integrates a decision-support mechanism based on the DM's preferences. The experimental results show its capacity to obtain consistent solutions in the Region of Interest. These solutions surpass in quality others obtained by alternative proposals taking into account the amount and impact of the supported projects.

Let us observe that the surrogate model not only reduces the dimensionality of the problem but also reduces the number of alternatives that result from the multiobjective optimization process, because it provides only nonoutranked solutions that are on the privileged zone of the known Pareto frontier. Comparisons between pair of alternatives are not so difficult as the number of objectives seems to suggest. Note that the objectives $N_{1}$ and $n_{\mathrm{sd} 1}$ are strongly more important than the remaining ones. So, the DM can apply a sort of lexicographic priority that allows a radical reduction of the set of solutions. Even though it is possible that ACO-SOP produces a number of portfolios that could not be easily handled by the DM, this situation can be addressed by the support of an expert analyst who, using a multicriteria decision method, provides a sufficiently small set of alternatives that can be handled by the DM.

Another point that is worthy of discussion is that the projects might involve objectives that are hard to judge, as is the case of the risk, because they are complex in both quantitative and qualitative elements. However, it is important to note that such cases are handled in the present approach through the ranking. Following this idea, let us note that this work addresses the special case of PSPSOP that has as the only available information the ranking of the projects and their costs and that it is the rank that integrates all the previous existing information about the criteria that characterized the projects. In other words, the instances of PSPSOP already capture information about criteria such as risk in the rank, and the methodology followed to do such integration is out of the scope of the present research. 
However, if it were the case that an alternative to solve the problem of integrating objectives into ranking should be proposed, it could be done through the family of ELECTRE methods [26], which can address qualitative and quantitative information in a unique aggregation model of preferences.

Finally, some situations that can be present in the instances of the special case of PSPSOP studied here are the existence of interdependence among criteria and/or projects. Fuzzy multicriteria methods derived from outranking approaches can handle interdependence among criteria and reflect it on the rank ordering of candidate projects (cf. [29]). So, the rank used by our proposal takes into account the criterion interdependence. On the other hand, the project interdependence remains a challenge for the proposed model; it is defined as part of the future work of this research to explore the possibility of integrating synergy negative and positive effects among projects through the incorporation of artificial projects that are related to their interdependence.

\section{Conflicts of Interest}

The authors declare that they have no conflicts of interest.

\section{Acknowledgments}

This work has been partially supported by the following CONACyT projects: (a) Fronteras de la Ciencias Project 1340; (b) Consolidation National Lab Project 280712; (c) Projects 236154 and 269890; (d) Project 280081, Red Temática para el Apoyo a la Decisión y Optimización Inteligente de Sistemas Complejos y de Gran Escala (OPTISAD), Universidad Autónoma de Nuevo León; and (e) Project 3058 from the program Cátedras CONACyT.

\section{References}

[1] D. Kleinmuntz, "Resource allocation decisions," in Advances in Decision Analysis: From Foundations to Applications, W. Edwards, D. von Winterfeldt, and R. F. Miles, Eds., pp. 400-410, Cambridge University Press, Cambridge, UK, 2007.

[2] A. Salo, J. Keisler, and A. Morton, "An invitation to portfolio decision analysis," in Portfolio Decision Analysis: Improved Methods for Resource Allocation, A. Salo, J. Keisler, and A. Morton, Eds., pp. 3-27, Springer Science \& Business Media, New York, NY, USA, 2011.

[3] M. A. Coffin and B. W. Taylor III, "Multiple criteria R\&D project selection and scheduling using fuzzy logic," Computers \& Operations Research, vol. 23, no. 3, pp. 207-220, 1996.

[4] J. Klapka, P. Piňos, and V. Ševčík, "Multicriterial Projects Selection," Intelligent Systems Reference Library, vol. 38, pp. 245261, 2013.

[5] C. Stummer and K. Heidenberger, "Interactive R\&D portfolio analysis with project interdependencies and time profiles of multiple objectives," IEEE Transactions on Engineering Management, vol. 50, no. 2, pp. 175-183, 2003.

[6] J. L. Ringuest, S. B. Graves, and R. H. Case, "Mean-Gini analysis in R\&D portfolio selection," European Journal of Operational Research, vol. 154, no. 1, pp. 157-169, 2004.

[7] C. Carlsson, R. Fullér, M. Heikkilä, and P. Majlender, "A fuzzy approach to $\mathrm{R} \& \mathrm{D}$ project portfolio selection," International
Journal of Approximate Reasoning, vol. 44, no. 2, pp. 93-105, 2007.

[8] X. Zhao, Y. Yang, G. Wu, J. Yang, and X. Xue, "A dynamic and fuzzy modeling approach for multiobjective rd project portfolio selection," Journal of Convergence Information Technology, vol. 7, no. 1, pp. 36-44, 2012.

[9] S. S. Bastiani, L. Cruz-Reyes, E. Fernandez, and C. Gomez, "Portfolio optimization from a set of preference ordered projects using an ant colony based multi-objective approach," International Journal of Computational Intelligence Systems, vol. 8, pp. 41-53, 2015.

[10] R. Cooper, S. Edgett, and E. Kleinschmidt, "Portfolio management for new product development: Results of an industry practices study," RéD Management, vol. 31, no. 4, pp. 361-380, 2001.

[11] E. Fernandez and R. Olmedo, "Public project portfolio optimization under a participatory paradigm," Applied Computational Intelligence and Soft Computing, vol. 2013, Article ID 891781, 13 pages, 2013.

[12] S. A. Gabriel, S. Kumar, J. Ordóñez, and A. Nasserian, "A multiobjective optimization model for project selection with probabilistic considerations," Socio-Economic Planning Sciences, vol. 40, no. 4, pp. 297-313, 2006.

[13] Y. Yang, S. Yang, and Y. Ma, "A literature review on decision making approaches for research and development project portfolio selection," in Proceedings of the CSAMSE Conference, 2012.

[14] E. Fernandez, L. F. Felix, and G. Mazcorro, "Multi-objective optimisation of an outranking model for public resources allocation on competing projects," International Journal of Operational Research, vol. 5, no. 2, pp. 190-210, 2009.

[15] L. Cruz, E. Fernandez, C. Gomez, G. Rivera, and F. Perez, "Many-objective portfolio optimization of interdependent projects with 'a priori' incorporation of decision-maker preferences," Applied Mathematics \& Information Sciences, vol. 8, no. 4, pp. 1517-1531, 2014.

[16] S. Altuntas and T. Dereli, "A novel approach based on DEMATEL method and patent citation analysis for prioritizing a portfolio of investment projects," Expert Systems with Applications, vol. 42, no. 3, pp. 1003-1012, 2015.

[17] M. Corazza, S. Funari, and R. Gusso, "An evolutionary approach to preference disaggregation in a MURAME-based creditworthiness problem," Applied Soft Computing, vol. 29, pp. 110-121, 2015.

[18] M. Collan and P. Luukka, "Evaluating R \& D projects as investments by using an overall ranking from four new fuzzy similarity measure based TOPSIS variants," IEEE Transactions on Fuzzy Systems, vol. 22, no. 3, pp. 505-515, 2014.

[19] G. Mavrotas, D. Diakoulaki, and Y. Caloghirou, "Project prioritization under policy restrictions. A combination of MCDA with 0-1 programming," European Journal of Operational Research, vol. 171, no. 1, pp. 296-308, 2006.

[20] G. A. Miller, "The magical number seven, plus or minus two: some limits on our capacity for processing information," The Psychological Review, vol. 63, no. 2, pp. 81-97, 1956.

[21] H. Ishibuchi, N. Tsukamoto, and Y. Nojima, "Evolutionary many-objective optimization: a short review," in Proceedings of the IEEE Congress on Evolutionary Computation (CEC '08), pp. 2419-2426, June 2008.

[22] D. W. Corne and J. D. Knowles, “Techniques for highly multiobjective optimisation: Some nondominated points are better than others," in Proceedings of the 9th Annual Genetic and 
Evolutionary Computation Conference, GECCO 2007, pp. 773780, New York, NY, USA, July 2007.

[23] J. López, C. A. Coello Coello, K. Oyama, and K. Fujii, "Alternative preference relation to deal with many-objective optimization problems," in Proceedings of the Evolutionary Multi-Criterion Optimization (EMO 2013), R. C. Purshouse, P. J. Fleming, C. M. Fonseca, S. Greco, and J. Shaw, Eds., Lecture Notes in Computer Science, pp. 291-306, Springer, 2013.

[24] R. C. Purshouse and P. J. Fleming, "Evolutionary manyobjective optimisation: an exploratory analysis," in Proceedings of the Congress on Evolutionary Computation (CEC '03), vol. 3, pp. 2066-2073, December 2003.

[25] E. Fernandez, E. Lopez, G. Mazcorro, R. Olmedo, and C. A. Coello Coello, "Application of the non-outranked sorting genetic algorithm to public project portfolio selection," Information Sciences, vol. 228, pp. 131-149, 2013.

[26] B. Roy, "The Outranking Approach and the Foundations of ELECTRE methods," in Readings in Multiple Criteria Decision Aid, C. Baena e Costa, Ed., vol. 31, pp. 133-161, Springer Science \& Business Media, Berlin, Germany, 1990.

[27] J. Figueira, V. Mousseau, and B. Roy, "Electre methods," in Multiple Criteria Decision Analysis: State of the Art Surveys, J. Figueira, S. Greco, and M. Ehrgott, Eds., vol. 78 of International Series in Operations Research \& Management Science, pp. 164195, Springer, New York, NY, USA, 2005.

[28] M. Dorigo and L. M. Gambardella, "Ant colony system: a cooperative learning approach to the traveling salesman problem," IEEE Transactions on Evolutionary Computation, vol. 1, no. 1, pp. 53-66, 1997.

[29] J. R. Figueira, S. Greco, and B. Roy, "ELECTRE methods with interaction between criteria: an extension of the concordance index," European Journal of Operational Research, vol. 199, no. 2, pp. 478-495, 2009. 

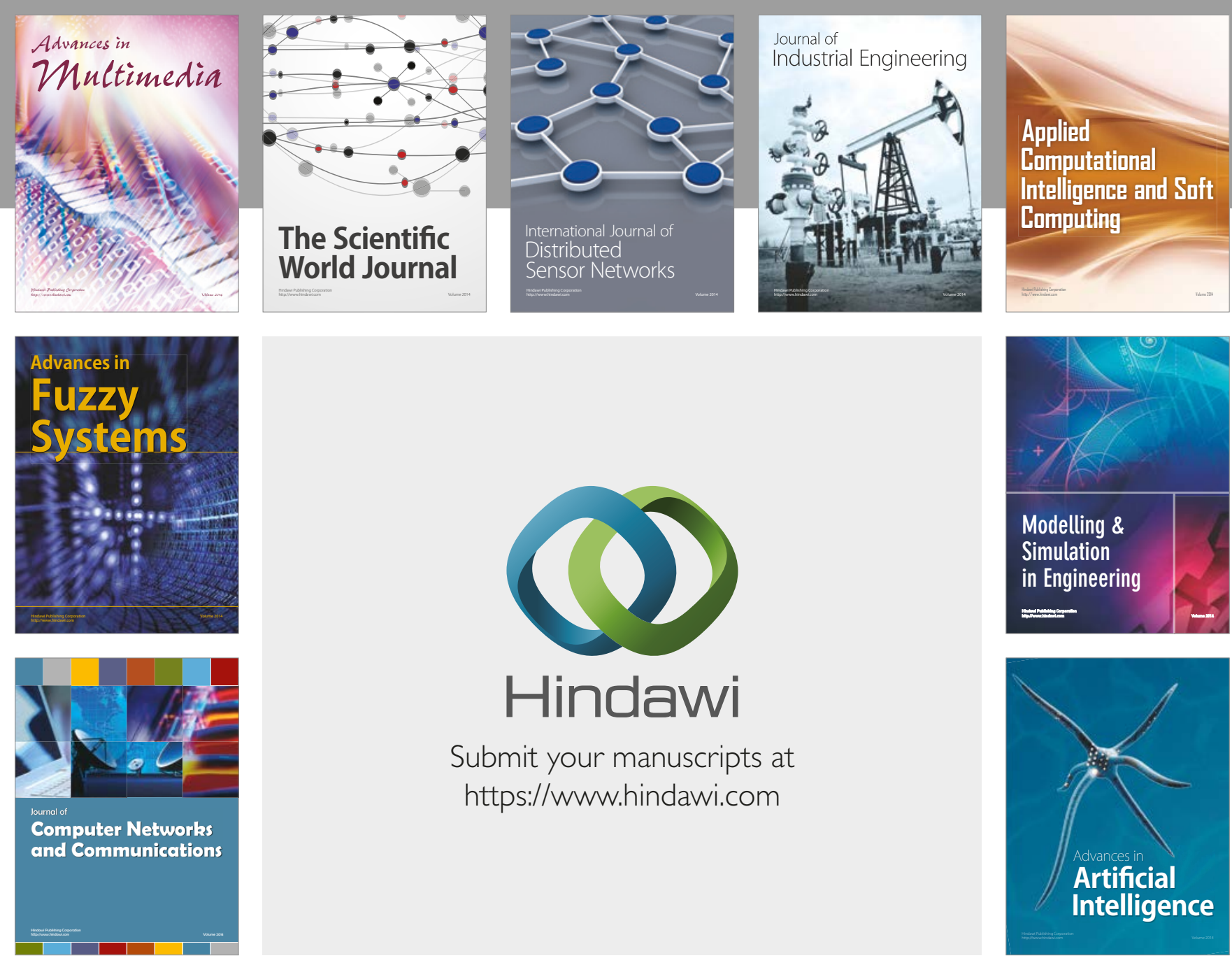

\section{Hindawi}

Submit your manuscripts at

https://www.hindawi.com
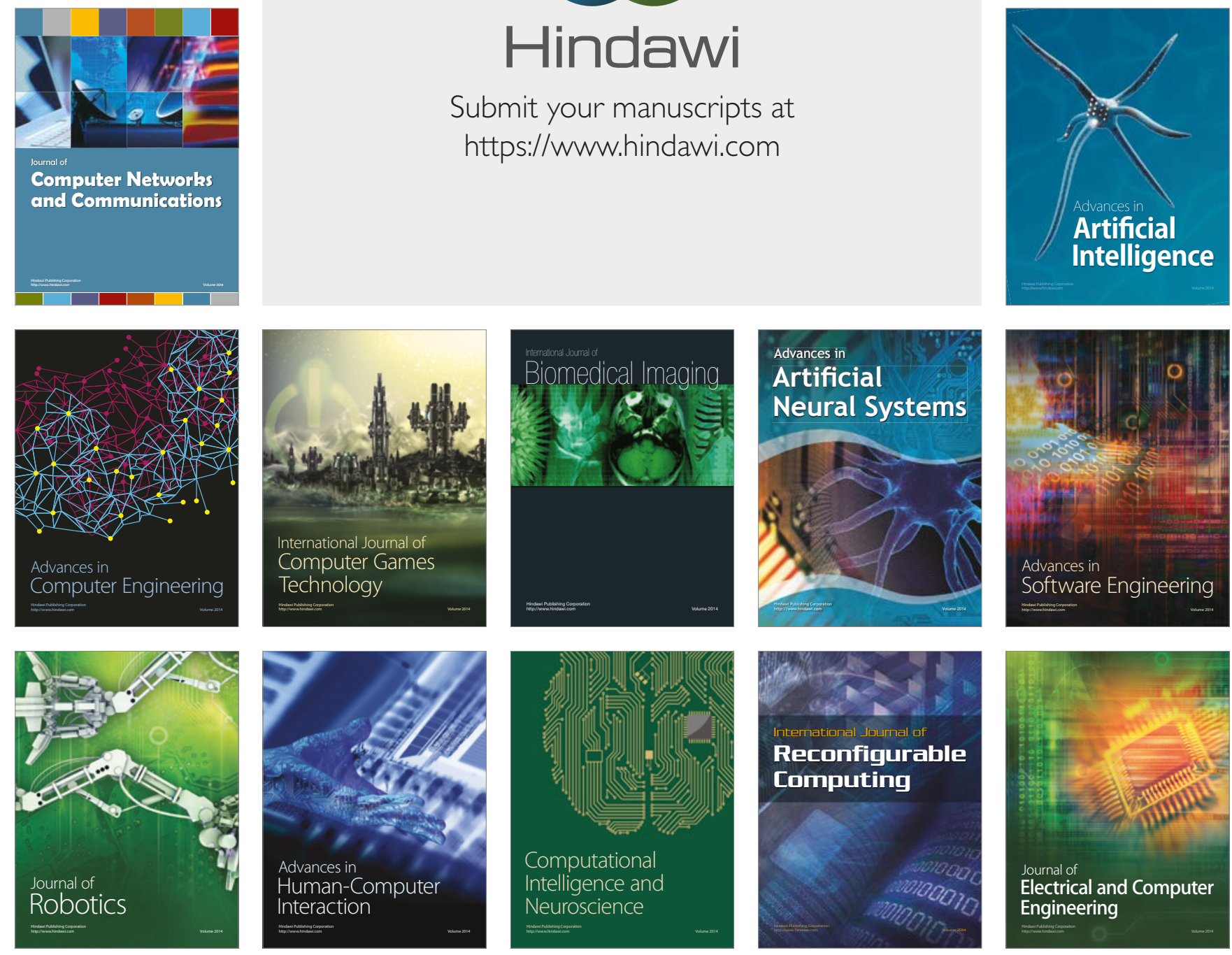